\title{
Scientific Issues with a Business Impact: In Vitro-In Vivo Workshop
}

he Workshop on Scientific Foundation and Applications for the Biopharmaceutics Classification System (BCS) and In Vitro-In Vivo Correlations (IVIVC) was a collaborative success. AAPS, Controlled Release Society (CRS), and the FDA co-sponsored the gathering, which took place April 14-16, 1997, in Arlington, VA.

The workshop clarified current scientific thinking on bioequivalence (BE) standards, identified gaps in knowledge, and determined needs for further research. Panelists addressed the basic physiological and pharmaceutical factors controlling drug absorption, controlled release dosage forms, and data analysis for Immediate Release (IR) and Extended Release (ER) products. In addition, they discussed regulatory considerations and the background and status of relevant FDA guidelines. The scientific bases for three guidances were presented. Two of the three guidances - the IR Dissolution Guidance and the Controlled Release Correlation Guidance-are both still in draft form; the BCS guidance is being developed by the FDA for release later this year.

\section{BCS: Membrane Permeability}

Gordon Amidon, Ph.D. stated that the panel agreed that human permeability should not be required to classify a drug. The work of Hans Lennernäs, Ph.D. on human permeability determination shows that the fraction dose $(\mathrm{Fa})$ absorbed in humans is generally proportional to permeability $(\mathrm{P})$ in humans, specifically for soluble drugs. Human permeability is also proportional to animal jejunal permeability, as evidenced in a variety of species. The important connection between $\mathrm{Fa}$ in humans and $\mathrm{P}$ in animals is where drug discovery and preclinical development data might affect future drug regulation. A scaling factor must be used for drugs with a carrier-mediated transport mechanism.

Reference compounds are extremely important to this process. The general consensus of the scientists on the panel was that the compounds should be structurally diverse so that
Reprinted from the AAPS Newsletter, June 1997 American Association of Pharmaceutical Scientists, Alexandria, VA

there is a high degree of confidence that a new chemical - a new molecular entity - is within the reference set in compound space. It may be possible to estimate or predict on the basis of permeability calculations; however, the research community is concerned about accuracy, so validation would still be needed.

Finally, the panel discussed $\mathrm{CaCo}-2$ tissue culture at length. They agreed that the same criteria should pertain to tissue culture that pertain to the in vitro and in vivo systems.

\section{BCS: Solubility and Drug Dissolution Considerations}

Ajaz Hussain, Ph.D. described this panel's focus on a highly soluble (HS), highly permeable (HP) class of drugs and the issues pertaining to that class. The panel agreed that for high solubility with a volume of $250 \mathrm{ml}$, the criteria for all $\mathrm{pHs}(1$ through 8 ) are probably very conservative. Second, for 85 percent dissolution in 15 minutes (the case A dissolution in SUPACIR), $0.1 \mathrm{~N} \mathrm{HCl}$ is also conservative. An additional $\mathrm{pH}$ of 6.8 should be considered normal for a dissolution test. Two possibilities are (1) to have two dissolution media tests for an HS, HP drug or (2) to include rapid dissolution in $6.8 \mathrm{pH}$.

The panel agreed that the dissolution rate is likely to be a good surrogate for Cmax. The HS, HP class will probably ensure extent of absorption at greater than 90 percent. High solubility is essential because the relationship between solubility and permeability is well defined for HS drugs.

\section{Gastrointestinal Variables}

Hans Lennernäs, Ph.D. listed recommendations from this panel. First, although disease states affect gastrointestinal variables, $\mathrm{BE}$ in control is acceptable. Second, in vitro testing is possible. Third, BE dissolution tests for lowsolubility drugs should include a $\mathrm{pH}$ of 1 to 8 , surfactant concentration between 0.5 and 2 percent (the panel

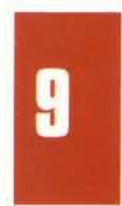




\section{Workshop....continued}

recommended putting it as low as possible), and an appropriate speed. Fourth, with physiological media for dissolution tests, composition and volume are related to the media, and hydrodynamics and duration also must be considered.

\section{Regulatory Considerations}

Henry Malinowski, Ph.D. presented this panel's summary highlights. Research at the universities of Maryland, Tennessee, Michigan, and Uppsala contributed policy to SUPAC-IR, SUPAC-ER, the correlation guidance, the gelatin capsule issue, and the BCS. Panelists generally agreed with the IR guidance and felt the profile comparison was useful. In the IR guidance, profile dissolution testing for IR products related to sameness. The panel expressed overall support for the BCS framework for using dissolution as a surrogate. One major reason is that it uses an in vivo characteristic, $\mathrm{P}$, and an in vitro characteristic, $\mathrm{S}$, in this framework, which makes strong scientific sense.

A question remains about the definition of high solubility and whether this characteristic should be related to the highest dose used or the highest strength available. The highest dose for defining high solubility relates more to clinical uses of drugs; however, in a BE study, the highest strength rather than the highest dose would normally be used. Further discussion is needed about how to best define high solubility.

\section{Modified Release Dosage Forms}

Jennifer Dressman, Ph.D. gave the summary report of this large panel. She mentioned a report by Ping Lee, Ph.D. on a wide range of mechanisms for modified release dosage forms. Patrick Sinko, Ph.D. indicated that release and dissolution may not always be synonymous in vivo, and this difference may not always be addressed by in vitro tests. Some key parameters to determine whether a dosage form has condition-dependent or independent release might include $\mathrm{pH}$ dependency, effective surfactants in the gastrointestinal (GI) tract, effective enzymes in the GI tract (in limited cases), and the effects of hydrodynamics. The drug properties, as well as the formulation, can determine whether the formulation will have condition-dependent performance. Some drugs have site-dependent permeability, particularly those with paracellular or active transport mechanisms. Linked to the latter would be sitedependent metabolism and efflux mechanisms. Site-dependent solubility of the drug is also possible. Modified-release dosage forms are more complicated than IR dosage forms because the excipients used in the formulation to produce a modified-release profile could induce condition-dependent release in the GI tract.

Lawrence Augsburger, Ph.D. gave a presentation illustrating that the University of Maryland modified-release results are consistent with the levels of testing suggested by SUPACMR and IVIVC guidances. The results were compiled separately from the data on which the guidances were based.

The panel suggested that incentives to establish an IVIVC could include fewer data requirements by the FDA later in the approval process. This might lead to wider specifications. Essential aspects of the IVIVC include the accuracy, precision, and predictability of the correlations; desirable aspects include the simplicity of the method, which relates to the robustness. Ideally, the correlation would be relevant to the physiology, both in the in vitro dissolution methodology and in the application of mathematical models. Most important, some potentially useful approaches include convolution, deconvolution, physiologically based pharmacokinetic models, compartmental models, and neural networks.

\section{Data Analysis for IR and ER Products}

Joseph Robinson, Ph.D., summarized this panel's discussion. For the most part, product quality is determined during design and development of the drug and dosage form. Flexibility in mathematical approach to correlation is needed during these processes. Understanding and using information about mechanisms are valuable; correlations can contribute to this understanding. The panel agreed that despite limits for some drugs, classification based on physical-chemical $\mathrm{x}$ Biological/biochemical issues require further research and should be incorporated into the process. The panel favored classification because it has the potential to reduce development and 
product support costs and regulatory burden.

The characteristics of dosage form effects on drug dissolution need to be considered in addition to drug properties (solubility, permeability). Once enough drugs have been studied with respect to solubility, permeability, or other relevant physical, chemical, or biological properties, the panel recommended the FDA identify a list of criteria that would allow categorization of drugs by relative biopharmaceutic risk. Biopharmaceutic risk equals the risk of adverse effects on patients without explicit studies to assess BE.

\section{Closing Remarks}

Dr. Amidon talked about the FDA's evolution toward openness in the guidance process and how the agency works with scientists and consumers. Cooperation and support from the FDA and the focus of Roger Williams, Ph.D. on pharmaceutical sciences and pharmaceutical product quality were cited as raising the quality of products for the marketplace. Dr. Amidon said that the support of industry in this meeting, with speakers openly discussing scientific issues that have a business impact, was very impressive. Dr. Robinson added that the International Pharmaceutical Federation (FIP) has set up a board of pharmaceutical sciences as a companion to the board of pharmacy.

AAPS press will soon release a publication entitled "Scientific Foundations for Regulating Drug Product Quality." The publication contains a summary from the Scientific Foundations and Applications for Biopharmaceutics Classification System (BCS) and In Vitro/In Vivo Correlations Workshop held April 14-16, 1997. The publication is scheduled to be released in September, 1997. For advance order information call AAPS at 703-548-3000. 